2006-1

\title{
Comparison of Three Electronic Speckle Pattern Shearing Interferometers using Photopolymer Holographic Optical Elements.
}

\author{
Emilia Mihaylova \\ Technological University Dublin, emilia.mihaylova@tudublin.ie \\ Izabela Naydenova \\ Technological University Dublin, izabela.naydenova@tudublin.ie \\ Vincent Toal \\ Technological University Dublin, vincent.toal@tudublin.ie
}

See next page for additional authors

Follow this and additional works at: https://arrow.tudublin.ie/cieocon2

Part of the Optics Commons

\section{Recommended Citation}

Mihaylova, E., Naydenova, I. \& Toal, V. (2006). Comparison of three electronic speckle pattern shearing interferometers using photopolymer holographic optical elements. SPIE proceedings of the International Conference on Holography, Optical Recording and Processing of Information, vol. 6252, pg. 466-471. doi:10.1117/12.677189

This Conference Paper is brought to you for free and open access by the Centre for Industrial and Engineering Optics at ARROW@TU Dublin. It has been accepted for inclusion in Conference Papers by an authorized administrator of ARROW@TU Dublin. For more information, please contact arrow.admin@tudublin.ie, aisling.coyne@tudublin.ie,gerard.connolly@tudublin.ie. Funder: Technological Sector Research Programme Strand III and the Arnold F. Graves Scholar Programme at Technological University Dublin

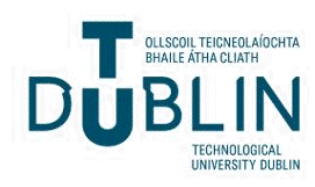




\section{Authors}

Emilia Mihaylova, Izabela Naydenova, Vincent Toal, and Suzanne Martin

This conference paper is available at ARROW@TU Dublin: https://arrow.tudublin.ie/cieocon2/23 
Dublin Institute of Technology

ARROW@DIT

2006-01-01

\section{Comparison of Three Electronic Speckle Pattern Shearing}

Emilia Mihaylova

Dublin Institute of Technology, emilia.mihaylova@dit.ie

Izabela Naydenova

Dublin Institute of Technology, izabela.naydenova@dit.ie

Vincent Toal

Dublin Institute of Technology, vincent.toal@dit.ie

Suzanne Martin

Dublin Institute of Technology, suzanne.martin@dit.ie

\section{Recommended Citation}

Mihaylova, E., Naydenova, I., Martin, S. Toal, V.: Comparison of three electronic speckle pattern shearing interferometers using photopolymer holographic optical elements. SPIE proceedings of the International Conference on Holography, Optical Recording and Processing of Information, Vol. 6252 pp 466-471. 2006. 


\title{
Comparison of three electronic speckle pattern shearing interferometers using photopolymer holographic optical elements
}

\author{
Emilia Mihaylova, Izabela Naydenova, Suzanne Martin, Vincent Toal \\ Centre for Industrial and Engineering Optics \\ Dublin Institute of Technology, Kevin Street, Dublin 8, Ireland \\ e-mail: emilia.mihaylova@dit.ie, izabela.naydenova@dit.ie,suzanne.martin@dit.ie, \\ vincent.toal@dit.ie
}

tel: 3531402 4702; fax: 35314024988

\begin{abstract}
Three electronic speckle pattern shearing interferometers (ESPSI) using photopolymer holographic gratings to produce the sheared image are presented. In the first ESPSI system two holographic gratings are used. The gratings are placed between the object and an imaging lens in front of the CCD camera. In the second ESPSI system one grating is used in combination with a sheet of ground glass. The sheared images on the ground glass are further imaged onto a CCD camera. In the third ESPSI system only one grating is used - it is placed in front of the object. The image and the sheared image are imaged onto the CCD camera, whose optical axis coincides with the normal to the object surface. The introduction of photopolymer holographic gratings in ESPSI systems gives the advantage of using high aperture optical elements at relatively low price. The systems are compared in terms of flexibility in their adjustment, sensitivity, suitability and limitations for different applications.
\end{abstract}

KEYWORDS: ESPSI, shearing interferometry, holographic grating, HOE

\section{INTRODUCTION}

Electronic speckle pattern shearing interferometry (ESPSI) enables direct measurements of displacement derivatives to be made with variable sensitivity ${ }^{1-8}$. A common approach to generate two sheared images of the object in ESPSI systems is to use a Michelson interferometric optical set-up. The images are generated via the two mirrors and the shear is introduced and controlled by tilting one of the mirrors. The idea of using a holographic grating for shearing of the two images in speckle shearing interferometry is not new ${ }^{5-9}$, but ESPSI using gratings to shear the image is an attractive alternative to the other shearographic systems $\mathrm{s}^{5-7}$ using gratings as it provides observation of real-time fringe formation and the possibility of phase-stepping analysis. We suggest new applications of photopolymer holographic gratings in three ESPSI optical set-ups. Self-processing acrylamide based photopolymer ${ }^{10}$ is used as a recording medium for recording holographic gratings. The optimized photopolymer material gives good diffraction efficiencies up to $94 \%$ for an exposure of $80 \mathrm{~mJ} / \mathrm{cm}^{2}$ and it performs well in the transmission mode of hologram recording. In the three ESPSI systems the photopolymer gratings are used to shear the image. Introducing photopolymer holographic gratings in ESPSI gives the advantage of using high aperture optical elements at relatively low price and makes the system compact. The three interferometric systems presented are simple, flexible and low cost.

\section{THEORY}

When two light waves interfere, the following equation ${ }^{11}$ relates their relative phase $\Phi$ at a point to their relative geometrical path difference $L$ :

$$
\Phi=\frac{2 \pi}{\lambda} n L-\beta
$$


where $\lambda$ is the wavelength of the laser light, $n$ is the refractive index of the medium through which the laser light is transmitted, and $\beta$ is a constant phase. The change in the relative phase $\Delta=\delta \Phi$ or phase change, which manifests as visible fringes, can be effected by an incremental change in any of the three parameters $\lambda, n$, and $L$. Thus,

$$
\Delta=\frac{\partial \Phi}{\partial \lambda} \delta \lambda+\frac{\partial \Phi}{\partial n} \delta n+\frac{\partial \Phi}{\partial L} \delta L=-\frac{2 \pi L n}{\lambda^{2}} \delta \lambda+\frac{2 \pi L}{\lambda} \delta n+\frac{2 \pi n}{\lambda} \delta L
$$

where $\delta \lambda, \delta n$, and $\delta L$ denote respectively, the incremental change in wavelength, in refractive index and the difference in relative geometrical path length of the interfering waves.

If the same wavelength is used and the test environment is still air, only the $\delta L$ term in Eq. (2) is nonzero, resulting in the following equation for the phase change ${ }^{12}$ :

$$
\Delta=\frac{2 \pi}{\lambda}[A \delta u+B \delta v+C \delta w]
$$

where $u, v$ and $w$ are the displacement components of the point $P^{\prime}(x+\Delta x, y+\Delta \mathrm{y}, z+\Delta z)$ relative to point $P(x, y, z)$ on the test surface, and $A, B$, and $C$ are sensitivity factors that are related to the optical arrangement. When a diffraction grating is used to introduce the shear the direction in which the system is sensitive can be precisely controlled by changing the orientation of the grating. If the grating introduces small image shearing $\Delta x$, the displacement terms in Eq. (3) can be expressed in terms of partial derivatives:

$$
\Delta=\frac{2 \pi}{\lambda}\left[A \frac{\partial u}{\partial x}+B \frac{\partial v}{\partial x}+C \frac{\partial w}{\partial x}\right] \Delta x
$$

In our case (Fig. 1, 3,5) the object beam lies in the $(\mathrm{x}, \mathrm{z})$ plane so there is no sensitivity in the y direction. The phase change is:

$$
\Delta=\frac{2 \pi}{\lambda}\left[A \frac{\partial u}{\partial x}+C \frac{\partial w}{\partial x}\right] \Delta x
$$

For a small image shear $\Delta x$ the phase difference $\Delta$ can be expressed as ${ }^{8}$ :

$$
\Delta=\frac{2 \pi}{\lambda}\left[\frac{\partial u}{\partial x} \sin \theta+\frac{\partial w}{\partial x}(1+\cos \theta)\right] \Delta x
$$

\section{EXPERIMENT}

\subsection{Photopolymer diffractive optical element}

Holographic gratings with spatial frequencies of 200 lines $/ \mathrm{mm}, 350$ lines $/ \mathrm{mm}$ and 500 lines $/ \mathrm{mm}$ were recorded using the second harmonic of a CW NdYVO 4 laser $(\lambda=532 \mathrm{~nm})$. The IEO acrylamide based photopolymer, which is selfdeveloping, was used as the photosensitive medium. The layers were approximately $100 \mu \mathrm{m}$ thick. Recording time and intensity were $20 \mathrm{~s}$ and $3.5 \mathrm{~mW} / \mathrm{cm}^{2}$ respectively. The diameter of these gratings is $40 \mathrm{~mm}$. Diffraction efficiency in the +1 order is $60 \%$. One of the advantages of this material is that characteristics such as diffraction efficiency, thickness (which controls selectivity), slant angle, diameter and reconstruction wavelength and angle can all be chosen to suit the specific application. The IEO photopolymer is characterized by low scattering and this is important when the imaging properties of the optical system are of concern.

\subsection{Experimental set-ups}

The schemes of the ESPSI systems using photopolymer holographic gratings are presented in Figures 1, 2 and 3. A Helium-Neon laser, with wavelength $633 \mathrm{~nm}$ and output power of $20 \mathrm{~mW}$, is used as the light source. The laser beam illuminates the object at an angle $\theta=30^{\circ}$ to the normal to the object surface. The intensities of the zero and the first order of diffraction were equalized by rotation of the gratings. The rotation of the gratings around the central axis, parallel to y-axis, leads to slight off-Bragg angular adjustment and decrease in the intensity of the first order thus offering the possibility for fine adjustment of both image and sheared image intensities. 


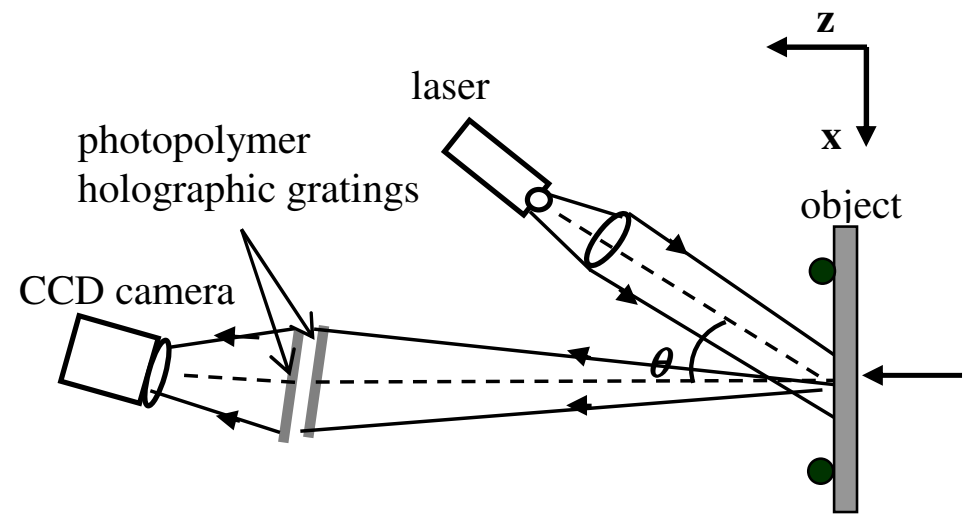

Fig. 1. An optical set-up of the ESPSI system with two photopolymer gratings ${ }^{13}$

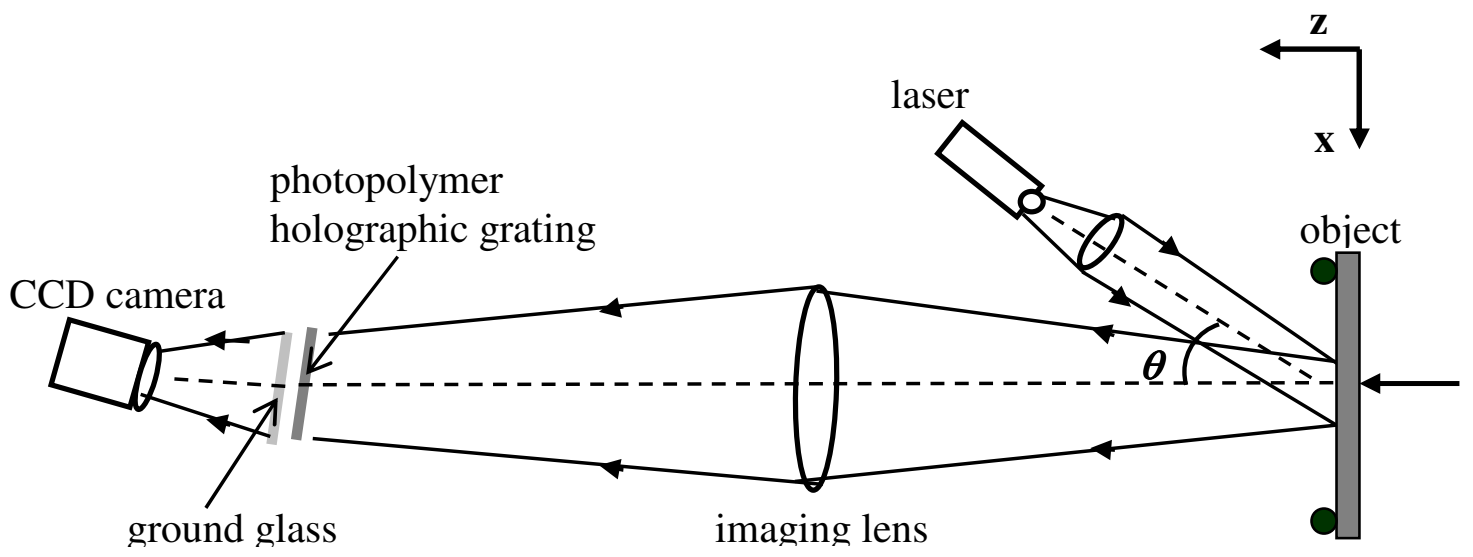

Fig. 2. An optical set-up of the ESPSI system with one photopolymer grating ${ }^{14}$ and ground glass

\subsection{Control of the shearing amount and shearing direction}

Easy control of the shearing amount is important because it determines the overall sensitivity of the systems. In the first ESPSI system the distance between the two gratings of the same frequencies controls the size of the shear (Fig. 4). In the second ESPSI system the distance between the grating and the ground glass can be used to control the amount of the shear (Fig. 5). In the third ESPSI system the distance between the grating and the object controls the size of the shear (Fig. 6). The increase of the amount of shear leads to an increase of the sensitivity of the system. When the distance between the two(? )elements is kept constant the shearing amount can be changed by utilizing diffraction gratings with different spatial frequencies. The higher is the spatial frequency the bigger is the amount of the shear. The spatial frequency of the gratings influences also other important parameters of the system such as the contrast of the obtained ESPSI fringes and the size of the field of view. 


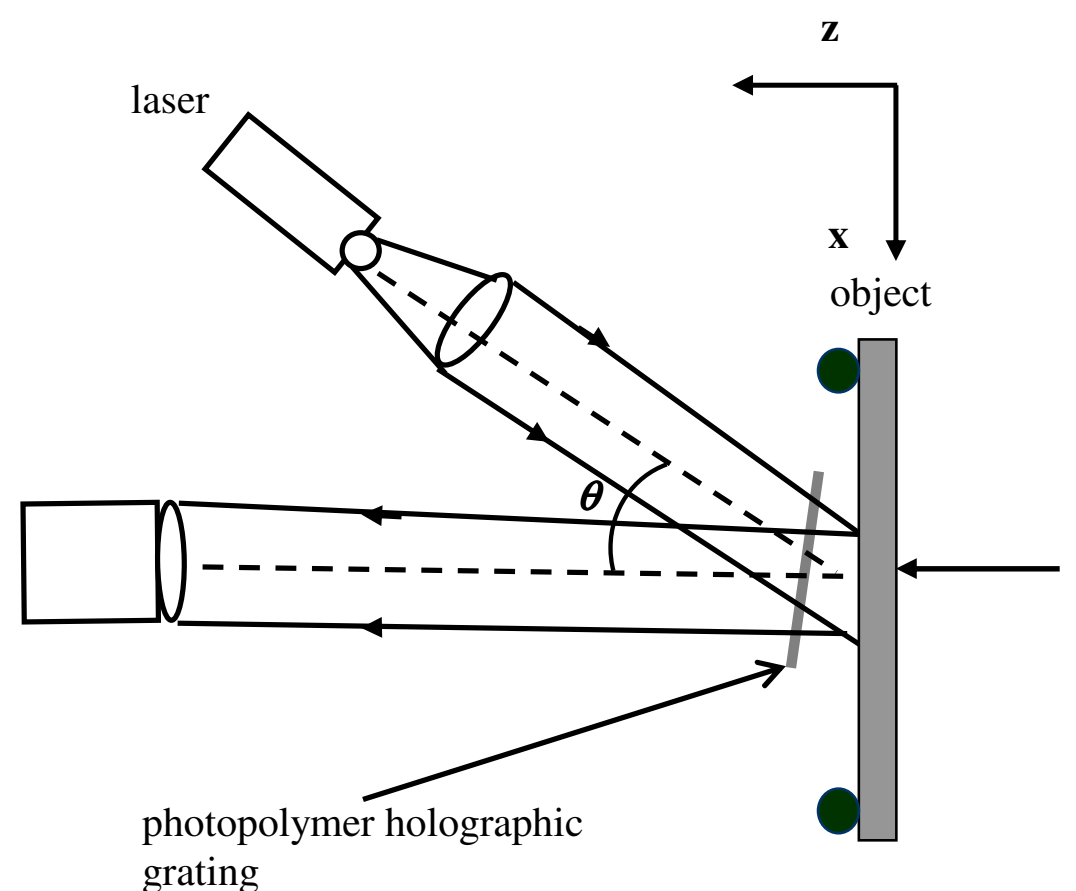

Fig. 3. An optical set-up of the ESPSI system with one photopolymer grating in front of the object

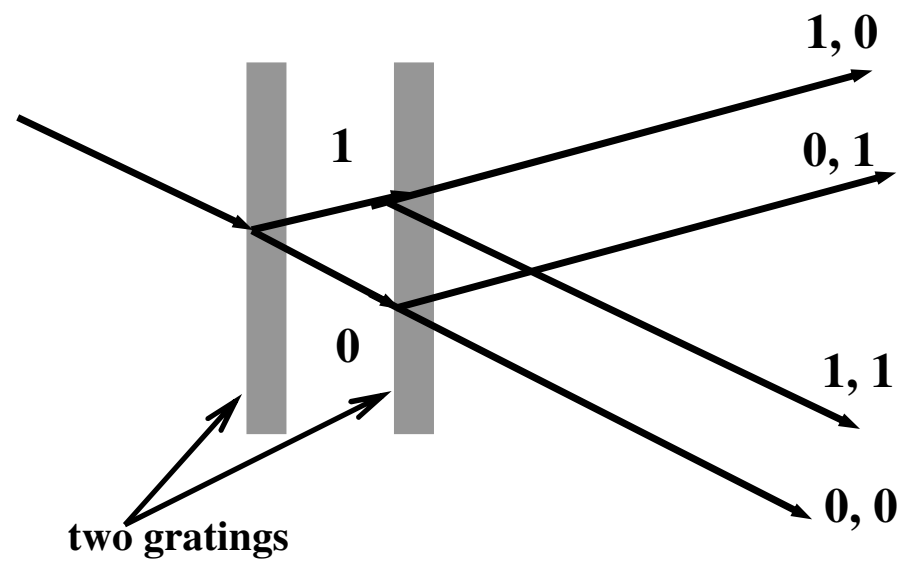

Fig. 4. Propagation of the beams through the shear introducing diffraction gratings 

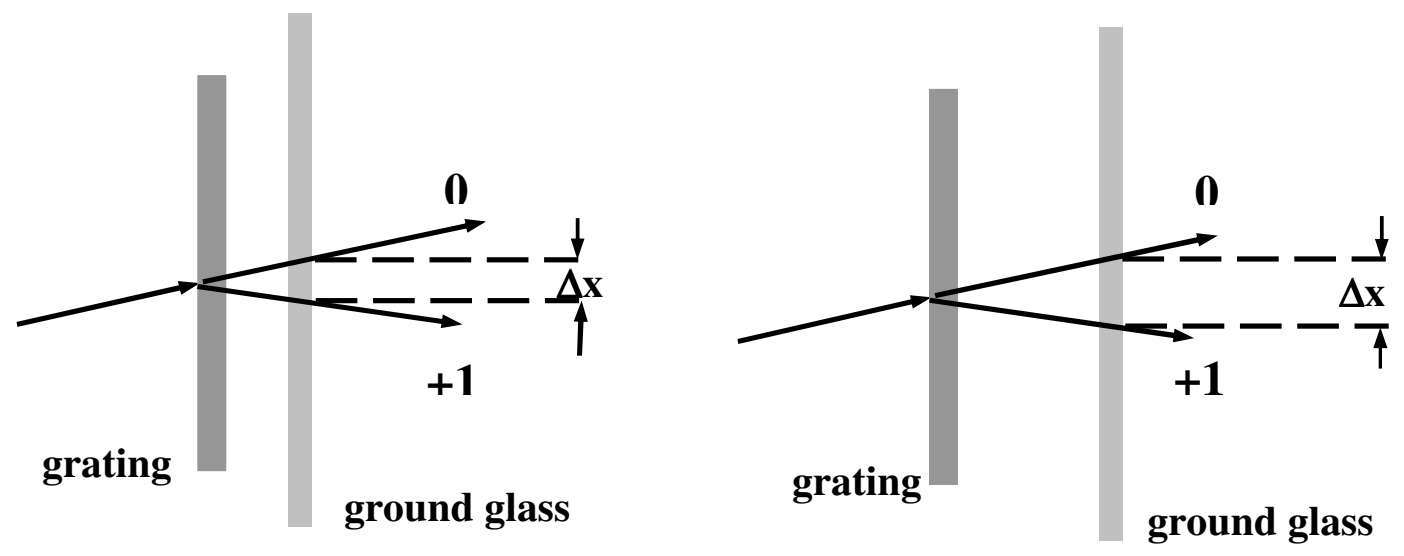

Fig. 5. Control of the shear in the ESPSI system with one photopolymer grating and ground glass

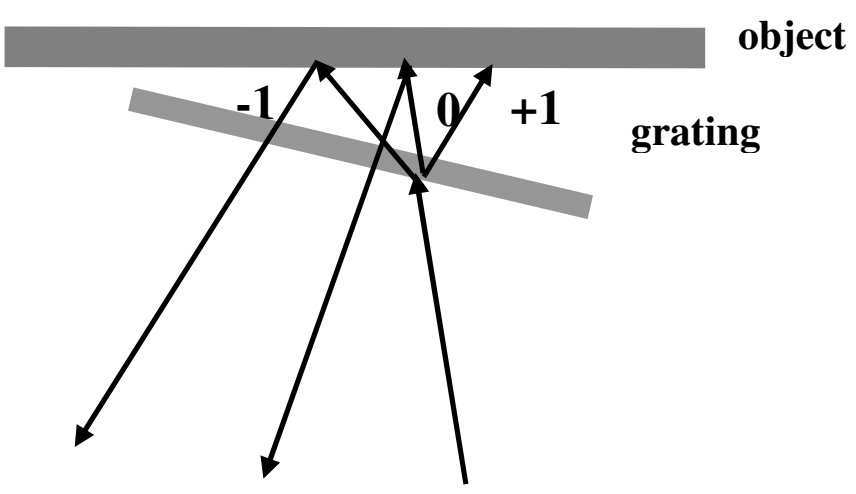

Fig. 6. Propagation of the beams through the shear introducing diffraction grating

\section{RESULTS AND DISCUSSION}

Figures 7, 8 and 9 show the results from the test of the three ESPSI systems using photopolymer holographic gratings to introduce the shear. All fringe patterns presented were recorded during cooling of an aluminium tin filled with hot water. A filter with a $3 \times 3$ window was used to reduce the speckle noise in the images. The fringe pattern contrast was estimated to be above $90 \%$.
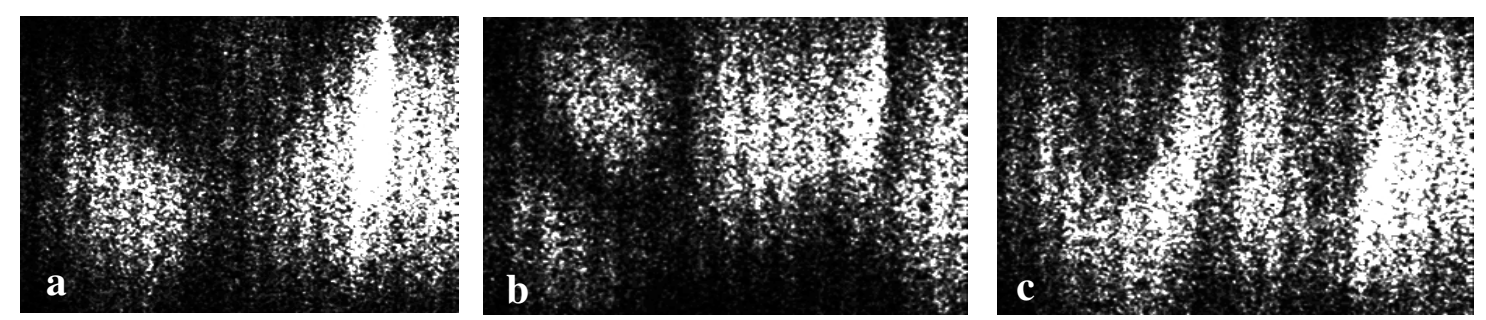

Fig. 7. ESPSI fringes in aluminium tin filled with hot water recorded during cooling with ESPSI system with two photopolymer holographic gratings: a) at the beginning of data acquisition; b) after $3 \mathrm{~s}$; c) after $6 \mathrm{~s}$. The field of view is $35 \mathrm{~mm} \times 30 \mathrm{~mm}$. 

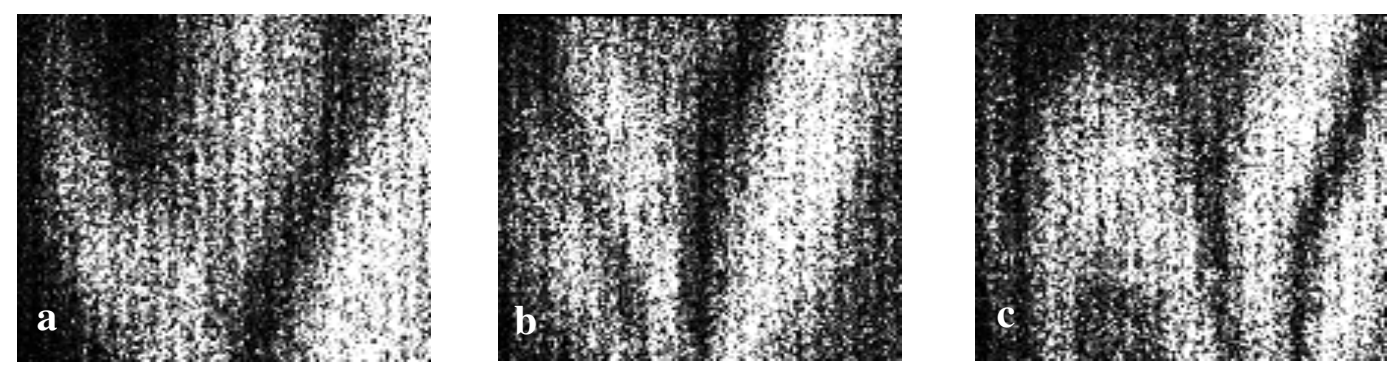

Fig. 8. ESPSI fringes in aluminium tin filled with hot water recorded during cooling with ESPSI system with one holographic grating and ground glass: a) at the beginning of data acquisition; b) after $3 \mathrm{~s}$; c) after $6 \mathrm{~s}$. The field of view is $19 \mathrm{~mm} \times 19 \mathrm{~mm}$.

The results of the comparison of the three ESPSI systems using photopolymer diffractive optical elements are presented in Table 1.

Table 1. Comparison of three electronic speckle pattern shearing interferometers using photopolymer holographic optical elements

\begin{tabular}{|c|c|c|c|c|c|c|}
\hline $\begin{array}{l}\text { ESPSI } \\
\text { system }\end{array}$ & $\begin{array}{l}\text { Control of } \\
\text { the shear }\end{array}$ & $\begin{array}{c}\text { Control of } \\
\text { the } \\
\text { intensity }\end{array}$ & $\begin{array}{c}\text { Changing } \\
\text { the } \\
\text { shearing } \\
\text { directions }\end{array}$ & $\begin{array}{l}\text { Maximum field of } \\
\text { view up to now }\end{array}$ & Compact-ness & Cost \\
\hline $\begin{array}{c}\text { One } \\
\text { grating } \\
\text { and } \\
\text { ground } \\
\text { glass }\end{array}$ & $\begin{array}{l}\text { by } \\
\text { changing } \\
\text { the } \\
\text { distance } \\
\text { between } \\
\text { the HG } \\
\text { and GG }\end{array}$ & $\begin{array}{l}\text { by rotating } \\
\text { the grating } \\
\text { around its } \\
\text { central axis }\end{array}$ & $\begin{array}{l}\text { by rotating } \\
\text { of the } \\
\text { grating } \\
\text { around its } \\
\text { normal }\end{array}$ & $60 \mathrm{~mm} \times 60 \mathrm{~mm}$ & $\begin{array}{c}5 \\
\text { components }\end{array}$ & low \\
\hline $\begin{array}{l}\text { Two } \\
\text { gratings }\end{array}$ & $\begin{array}{l}\text { by } \\
\text { changing } \\
\text { the } \\
\text { distance } \\
\text { between } \\
\text { the two } \\
\text { HGs }\end{array}$ & $\begin{array}{l}\text { by rotating } \\
\text { one of the } 2 \\
\text { HGs around } \\
\text { the central } \\
\text { axis }\end{array}$ & $\begin{array}{l}\text { by rotating } \\
\text { of the two } \\
\text { gratings } \\
\text { around the } \\
\text { normal }\end{array}$ & $35 \mathrm{~mm} \times 35 \mathrm{~mm}$ & $\begin{array}{c}4 \\
\text { components }\end{array}$ & low \\
\hline $\begin{array}{c}\text { One } \\
\text { grating in } \\
\text { front of the } \\
\text { object }\end{array}$ & $\begin{array}{l}\text { by } \\
\text { changing } \\
\text { the } \\
\text { distance } \\
\text { between } \\
\text { the HG } \\
\text { and the } \\
\text { object }\end{array}$ & $\begin{array}{l}\text { by rotating } \\
\text { the grating } \\
\text { around its } \\
\text { central axis }\end{array}$ & $\begin{array}{l}\text { by rotating } \\
\text { of the } \\
\text { grating } \\
\text { around its } \\
\text { normal }\end{array}$ & $30 \mathrm{~mm} \times 30 \mathrm{~mm}$ & $\begin{array}{c}3 \\
\text { components }\end{array}$ & low \\
\hline
\end{tabular}



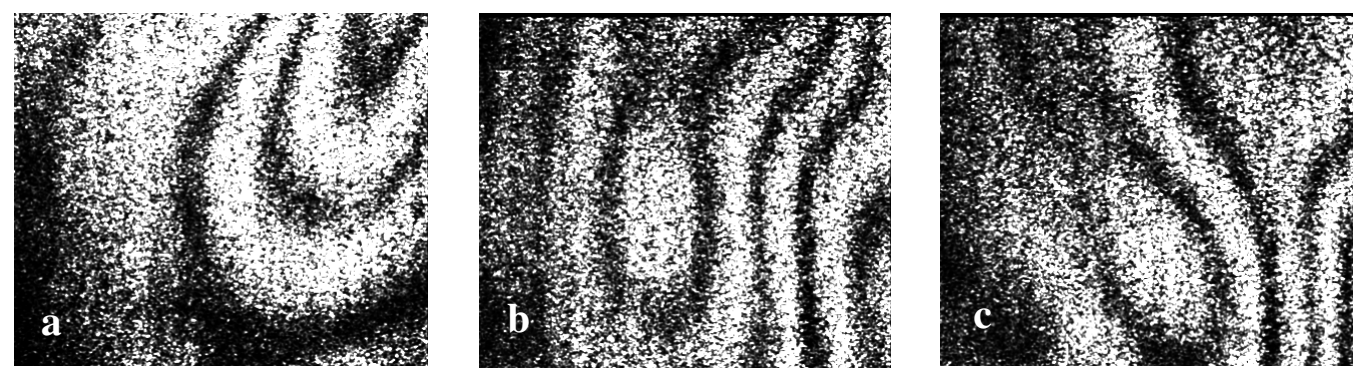

Fig. 9. ESPSI fringes in aluminium tin filled with hot water recorded during cooling with ESPSI system with one grating in front of the object: a) at the beginning of data acquisition; b) after $3 \mathrm{~s}$; c) after $6 \mathrm{~s}$. The field of view is $16 \mathrm{~mm} \times 14 \mathrm{~mm}$.

\section{CONCLUSIONS}

New applications of photopolymer diffractive optical elements in electronic speckle pattern shearing interferometry are presented. Three optical set-ups for electronic speckle pattern shearing interferometry using photopolymer gratings are presented. Holographic gratings are recorded using an acrylamide based photopolymer material.

The ESPSI systems using diffraction gratings to shear the image have some advantages compared to the commercially available systems based on a combination of a beam splitter and two mirrors (modified Michelson interferometer). They are simple and flexible and offer a simple way to introduce discrete shear steps between two images. Another advantage of these systems is that it is easy to change the shearing direction by rotating of the grating(s) around its normal. Advantages include also the low cost of these systems and the potential to use large apertures.

It is easy to apply the ESPSI systems using HOE to shear the image in a phase-shifting mode. Mounting the glass plate or the HOE on a piezoelectric transducer (PZT) allows the introduction of a known phase shift.

\section{ACKNOWLEDGEMENTS}

Acknowledgements_are made to Technological Sector Research Programme Strand III supported by the Irish Government. Emilia Mihaylova and Izabela Naydenova would like to thank the Arnold F. Graves Scholar Programme at Dublin Institute of Technology.

\section{REFERENCES}

1. C. Joenathan C. and Torroba R., "Simple electronic speckle shearing pattern interferometer", Opt. Lett. 15 (20), 1159-1161 (1990).

2. R. S. Sirohi, "Speckle methods in experimental mechanics", in Speckle Metrology, Ed. R. S. Sirohi, Mercel Dekker, New York (1993).

3. Y. M. He, C. J. Tay, H. M. Shang, "Digital phase-shifting shearography for slope measurement", Opt. Eng. 38 (9), 1586-1590 (1999).

4. H. M. Shang, Y. Y. Hung, W. D. Luo, F. Chen, "Surface profiling using shearography", Opt. Eng. 39(1), 23-31 (2000).

5. P. Hariharan, "Speckle-shearing interferometry: a simple optical system", Appl. Opt. 14 (11), 2563 (1975).

6. Y. Iwahashi, K. Iwata, and R. Nagata, "Single-aperture speckle shearing interferometry with a single grating", Appl. Opt. 23 (2), 247-249 (1984).

7. C. Joenathan and R. S. Sirohi, "Holographic gratings in speckle shearing interferometry", Appl. Opt. 24 (17), 2750-2751 (1985).

8. H. Rabal, R. Henao, R. Torroba, "Digital speckle pattern shearing interferometry using diffraction gratings", Optics Comm. 126, 191-196 (1996). 
9. C. Joenathan, L. Bürkle, "Elecktronic speckle pattern shearing interferometer using holographic gratings", Opt. Eng. 36 (9), 2473-2477 (1997).

10. S. Martin, P. Leclère, V. Toal, Y. Renotte and Y. Lion, "Characterisation of acrylamide-based photopolymer holographic recording material”, Opt. Eng. 32 (12), 3942 - 3946 (1994).

11. C. M. Vest, Holographic Interferometry, John Wiley, New York (1979).

12. Y. Y. Hung and C. Y. Liang, "Image shearing camera for direct measurement of surface-strains", Appl. Opt. 10(7), 1046-1050 (1979).

13. Emilia Mihaylova, Izabela Naydenova, Hosam Sherif, Suzanne Martin, Vincent Toal, "Application of photopolymer holographic gratings in electronic speckle pattern shearing interferometry", Proceedings of SPIE 5249, 318-326 (2003).

14. Emilia Mihaylova, Izabela Naydenova, Suzanne Martin, Vincent Toal, "Electronic speckle pattern shearing interferometer with a photopolymer holographic grating", Applied Optics 43 (12), 2439 - 2442 (2004). 